\title{
Microwave emission from dust revisited (Research Note)
}

\author{
A. P. Jones \\ Institut d'Astrophysique Spatial, Université Paris Sud (CNRS), Bâtiment 121, 91405 Orsay Cedex, France \\ e-mail: Anthony.Jones@ias.u-psud.fr
}

Received 16 July 2008 / Accepted 31 July 2009

\begin{abstract}
Context. The origin of the anomalous microwave emission is not yet identified but it may be associated with (spinning) dust. Aims. We point out that the emission from low-energy, solid-state structural transitions, apparent over a range of dust temperatures, and particularly important at low dust temperatures $\left(T_{\mathrm{d}}<30 \mathrm{~K}\right)$, could provide an origin for the excess microwave emission. Methods. The physics of two-level systems (TLS) within amorphous materials is applied within the context of interstellar dust. Results. TLS systems, which are thought to be at the origin of the temperature-dependent emissivity of interstellar dust, should reveal their existence at long wavelengths in the emission from dust.

Conclusions. Low-energy, structural transitions in amorphous interstellar grains could make a contribution to the anomalous microwave emission, which should correlate with the emission from dust components with temperatures less than about $30 \mathrm{~K}$.
\end{abstract}

Key words. dust, extinction - ISM: lines and bands

\section{Introduction}

Experimental evidence shows that the physical properties of amorphous silicate, oxide and carbonaceous materials show anomalous behaviour at low temperatures. For example, amorphous silicate material heat capacities may be significantly enhanced at very low temperatures (e.g. less than $1 \mathrm{~K}$ Zeller \& Pohl 1971), an effect that can still be important up to temperatures of the order of $30 \mathrm{~K}$ or more. This is related to the observation that the FIR to sub-mm emissivity index of interstellar dust analogue materials, in the laboratory, has been shown to be temperaturedependent (Agladze et al. 1995; Mennella et al. 1998; Boudet et al. 2005). Cold interstellar grains should therefore manifest some of these properties in their long wavelength emission when their temperatures fall within the range of interest. Such effects have already been observed in the dust emission at FIR to sub$\mathrm{mm}$ wavelengths in a variety of galactic regions and indicate significant differences compared to the standard dust model results (e.g., Ristorcelli et al. 1998; Bernard et al. 1999; Stepnik et al. 2003; Dupac et al. 2001, 2002, 2003). These data show an enhancement in the dust emissivity index for dust temperatures less than about $30 \mathrm{~K}$.

The anomalous physical properties of amorphous materials at low temperatures have been extensively reviewed by Phillips (1987). From the review by Phillips it is evident that the unexpected thermal properties of amorphous materials arise from the presence of excitations that can scatter phonons and thus contribute to an enhancement in the heat capacity at low temperatures. In particular, it appears that the two-level tunnelling systems provide a good model for the origin of these lowtemperature physical effects (Phillips 1987; Meny et al. 2007) and for the interpretation of the temperature-dependent emissivities seen at FIR to sub-mm wavelengths in laboratory and astronomical data (Agladze et al. 1995; Mennella et al. 1998;
Jones 2001, 2002; Boudet et al. 2005) and the observed microwave emission excess (Peng 2007). In the two-level systems the tunnelling of atoms between the two minima gives rise to the small energy splittings needed. The number of atoms contributing to these transitions at low temperatures turns out to be only a small fraction of the total. In particular, we note that the energy splitting of the two-level systems, of the order of $10^{-4} \mathrm{eV}$ (Phillips 1987), is equivalent to $1.2 \mathrm{~K}, 1.24 \mathrm{~cm}$ or $24.2 \mathrm{GHz}$. The latter figure is of interest because it puts the two-level system energies in the wavelength range of the observed anomalous microwave emission that extends from $\sim 100 \mathrm{GHz}$ to lower frequencies and that peaks in the $10-40 \mathrm{GHz}$ region.

The aim of this Research Note is to highlight lowtemperature effects in amorphous silicate, oxide and carbonaceous materials and to show that there could be a significant link between them and the observed anomalous microwave emission. A link would imply an origin for the anomalous microwave emission in relatively cold interstellar grains and would be an alternative to the spinning dust hypothesis (e.g., Draine \& Lazarian 1998a,b), magnetic dipole emission (Draine \& Lazarian 1999) or PAH rotational emission (Ysard \& Verstraete 2008).

\section{The dust emission at microwave wavelengths}

The total dust emission at FIR to sub-mm wavelengths can be modelled as a sum of the dust infrared vibrational emission (the wavelength-dependent, emissivity-modified black-body emission) and that of the two-level tunnelling transitions as follows:

$\frac{j_{v}}{n_{\mathrm{H}}}=A\left[\kappa_{v}+\frac{3}{4 a \rho} f_{\mathrm{TLS}} g_{v}\right] B_{v}\left(T_{\mathrm{d}}\right) \quad \mathrm{Jy} \mathrm{sr}^{-1} \mathrm{H}^{-1}$

where $\kappa_{v}$ is the dust mass absorption coefficient, $f_{\text {TLS }}$ is the fraction of the dust mass (i.e., fraction of the atoms) contributing 


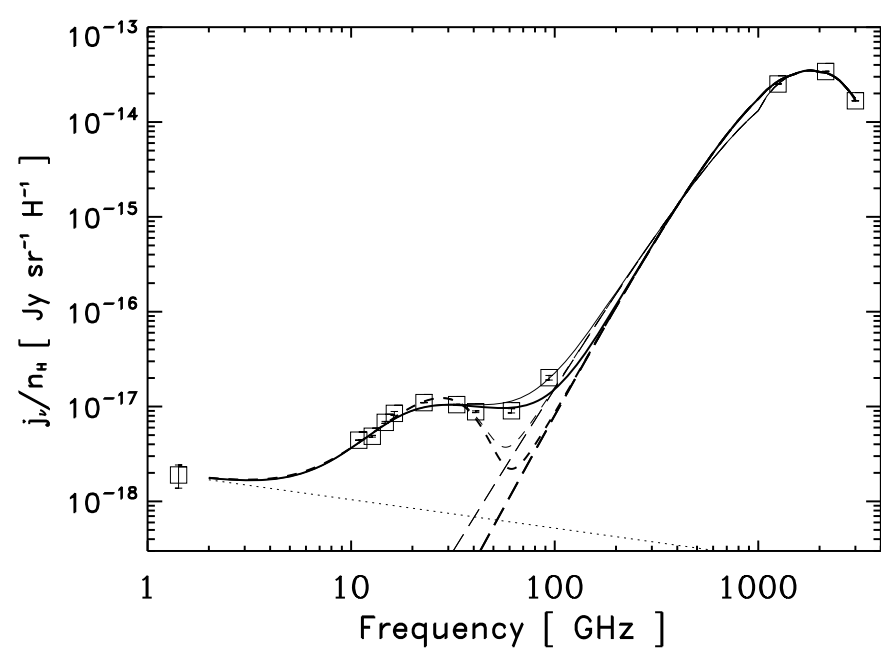

Fig. 1. The vibrational emision (long-dashed lines) of amorphous silicate dust (a-Sil, $T_{\mathrm{d}}=17.5 \mathrm{~K}$, thick lines) and hydrogenated amorphous carbon dust (a-C:H, $T_{\mathrm{d}}=20.0 \mathrm{~K}$, thin lines). Superimposed on the dust emission at frequencies less than $\approx 200 \mathrm{GHz}$ are the assumed TLS emission profiles for Lorentzian (solid lines) and Gaussian (short-dashed lines) band profiles, $g_{v}$. The observational data points and the synchrotron contribution (dotted line) are taken from Watson et al. (2005).

to the TLS states, $B_{v}\left(T_{\mathrm{d}}\right)$ is the Planck function and $g_{v}$ is the adopted band profile for the two-level tunnelling states. Here we adopt $4 \pi v j_{v} / n_{\mathrm{H}}=9.4 \times 10^{-24} \mathrm{erg} \mathrm{s}^{-1} \mathrm{H}^{-1}$ to fit the peak of the dust vibrational emission at $140 \mu \mathrm{m}$ (following the formalism of Draine \& Lazarian 1998a) corresponding to $A=3.5 \times 10^{-14}$. The factor $3 /(4 a \rho)$ converts the TLS contribution to an effective mass absorption coefficient, i.e., $\kappa_{\mathrm{TLS}}=3 f_{\mathrm{TLS}} g_{v} /(4 a \rho)$. The exact form of the TLS transition profiles is unclear until such time as detailed laboratory data on interstellar dust analogues at low temperatures and long wavelengths becomes available. Here we adopt Lorentzian and Gaussian distributions for illustrative purposes. For the Lorentzian (Gaussian) profile we adopt $F W H M=30 \mathrm{GHz}(35 \mathrm{GHz})$.

In order to explain the observed low-temperature properties it appears that the density of states that would be required to explain them is rather low and of the order of $\simeq 10^{46} \mathrm{~J} \mathrm{~m}^{-3} \equiv$ $1.6 \mathrm{eV} \mathrm{nm}^{-3}$ or, equivalently, the fractional number of the contributing atoms, $f_{\mathrm{TLS}}$, is of the order of $10^{-4}$ (Phillips 1987).

If we adopt some typical values for the TLS profiledetermining parameters in Eq. (1), i.e., the contributing dust mass fraction $f_{\text {TLS }}=0.45 \times 10^{-4}$ for amorphous silicate and $1.20 \times 10^{-4}$ for hydrogenated amorphous carbon and dust temperatures of $T_{\mathrm{d}}=17.5 \mathrm{~K}$ and $20.0 \mathrm{~K}$, respectively, we find a good fit to the observed microwave emission excess. The model results are shown in Fig. 1. For the dust optical properties we assume those of "astronomical" silicate (Draine \& Lee 1984) and amorphous hydrogenated hydrocarbon (BE sample from Rouleau \& Martin 1991) and a grain radius $a=0.1 \mu \mathrm{m}$. The dust absorptivity is expressed in terms of the mass absorption coefficient, $\kappa_{v}$, which is proportional to the the grain size-independent quantity $Q_{\text {abs }} / a$ at these long wavelengths. The TLS process is measured on bulk samples in the laboratory and we therefore do not expect a significant size-dependence for the TLS process. However, this has yet to be shown by experiment.

This model reproduces the form of the microwave emission excess with some reasonable assumptions about the TLS parameters and some typical dust parameters. Clearly the
Lorentzian profile gives a rather good match to the observational data.

\section{Discussion and conclusions}

If this rather simple idea for the origin of the observed microwave emission excess were to prove correct then it would imply that the observed anomalous emission should correlate with the amorphous dust (carbonaceous or silicate/oxide) emission for temperatures less than about $30 \mathrm{~K}$.

To date the anomalous microwave emission does not seem to be unambiguously correlated with any particular dust component. Recent observations by Dickinson et al. (2006), which searched for a microwave emission excess in an $\mathrm{H}$ II region, puts an upper limit on the dust emission at $31 \mathrm{GHz}$ and appears to be inconsistent with the spinning dust model. However, this work also puts an upper limit of $2 \%$ on the polarised emission. The latter would tend to perhaps argue against large grains as the origin of the microwave excess. However, microwave emission from the two-level systems should be important for all amorphous dust materials at low temperatures. This primary requirement, i.e., for it be amorphous and have $T_{\text {dust }}<30 \mathrm{~K}$, would seem to indicate that a rather large fraction of the dust mass may be involved. Given that stochastically-heated small grains spend a significant fraction of their time at temperatures considerably less than their peak temperatures they may also contribute significantly to the observed excess microwave emission. Therefore, and because of the likely general nature of the TLS behaviour, it may be difficult to tie the origin of the excess emission to a particular dust grain size or composition.

Until detailed modelling of the microwave emission from cold dust at low temperatures $\left(T_{\text {dust }}<30 \mathrm{~K}\right)$ has been performed, in conjunction with new laboratory data, it may be premature to rule out any specific origin for the observed microwave excess from any particular grain component.

\section{References}

Agladze, N. I., Sievers, A. J., \& Jones, S. A. 1995, ApJ, 462, 1026 Bernard, J.-P., Abergel, A., Ristorcelli, I., et al. 1999, A\&A, 347, 640 Boudet, N., Mutschke, H., Nayral, C., et al. 2005, ApJ, 633, 272 Dickinson, C., Cassus, S., Pineda, J. L., et al. 2006, ApJ, 643, L111 Draine, B. T., \& Lazarian, A. 1998a, ApJ, 494, L19

Draine, B. T., \& Lazarian, A. 1998b, ApJ, 508, 157

Draine, B. T., \& Lazarian, A. 1999, ApJ, 512, 740

Draine, B. T., \& Lee, H. K. 1984, ApJ, 285, 89

Dupac, X., Giard, M., Bernard, J.-P., et al. 2001, ApJ, 553, 604 Dupac, X., Giard, M., Bernard, J.-P., et al. 2002, A\&A, 392, 691

Dupac, X., Bernard, J.-P., Boudet, N., et al. 2003, A\&A, 404, L11

Jones, A. P. 2001, in From Darkness to Light, Origin and Evolution of Young Stellar Clusters, ed. T. Montmerle, \& P. André, ASP Conf. Ser., San Francisco, 243, 37

Jones, A. P. 2002, in Infrared and Submillimeter Space Astronomy, ed. M. Giard, J. P. Bernard, A. Klotz, \& I. Ristorcelli (Les Ulis: EDP Sciences), EAS Publ. Ser., 4, 37

Mennella, V., Brucato, J. R., Colangeli, L., et al. 1998, ApJ, 496, 1058

Meny, C., Gromov, V., \& Boudet, N. 2007, A\&A, 468, 171

Peng, Y. 2007, Masters Degree Thesis, Université Paul Sabatier, Toulouse III, http://epubl.1tu.se/1653-0187/2007/074/ LTU-PB-EX-07074-SE.pdf

Phillips, W. A. 1987, Rep. Prog. Phys., 50, 1657

Ristorcelli, I., Serra, G., \& Lamarre, J. M. 1998, ApJ, 496, 267

Rouleau, F., \& Martin, P. G. 1991, ApJ, 377, 526

Stepnik, B., Abergel, A., Bernard, J.-P., et al. 2003, A\&A, 398, 551

Watson, R. A., Rebolo, R., Rubiño-Martin, J. A., et al. 2005, ApJ, 624, L89

Ysard, N., \& Verstraete, L. 2009, A\&A, submitted

Zeller, R. C., \& Pohl, R. O. 1971, Phys. Rev. B, 4, 2029 